\title{
Pulse Nanosecond Emitter for High Resolution Optical Radars
}

\author{
Alexey Filimonov, \\ Peter the Great St. Petersburg \\ Polytechnic University, \\ St. Petersburg, Russia \\ filimonov@rphf.spbstu.ru \\ Sergey Vainshtein, \\ CAS Group, \\ Oulu University, \\ Oulu, Finland \\ vais@ee.oulu.fi
}

\author{
Aleksander Fotiadi, \\ Peter the Great St. Petersburg \\ Polytechnic University, \\ St. Petersburg, Russia \\ fotiadi@rphf.spbstu.ru \\ Valery Zemlyakov, \\ National research university of \\ electronic technology, \\ Zelenograd, Moscow, Russia \\ vzml@rambler.ru
}

\author{
Galina Politova, \\ Baikov Institute of Metallurgyand \\ Materials Science, \\ Moscow, Russia \\ gpolitova@gmail.com
}

\begin{abstract}
The aim of the work is to create a miniature pulse optical emitter on an infrared laser diode with a pulse duration of the order of $1 \mathrm{~ns}$ and a pulse power of more than $30 \mathrm{~W}$ based on the effect of high-efficiency avalanche switching in bipolar silicon transistors. An optical emitter that can improve the accuracy of optical radar operation up to 10 times and which is a record indicator has been developed. The ways of increasing the radiation power, increasing the repetition rate of pulses (necessary to improve the accuracy of the radar), and searching for ways to transition to the subnanosecond region are considered.
\end{abstract}

Keywords - optical radar, high-speed switch, current driver, miniature assembly, high pulse power

\section{INTRODUCTION}

Modern long-range optical lidars operating in the nearinfrared range use pulse-modulated transmitters on laser diodes capable of emitting optical pulses with a peak power of up to $40 \mathrm{~W}$ for a duration of 3-10 ns. The term lidar stands for Light Detection and Ranging - LIDAR refers to ranging systems operating in the optical range and using a gas laser or a laser diode as a radiation source. The frequency range of the receiving channel today is generally more than $300 \mathrm{MHz}$, and, therefore, the duration of pulses more than $3 \mathrm{~ns}$ is a significant limitation for such tasks as increasing the accuracy of optical ranging.

The performance of modern high-current drivers is usually limited by the switching time of switch components such as field effect transistors and avalanche switches. In this paper, it is shown that the type of transistor, operating voltage, parasitic inductance in a miniature arrangement and also the method of mounting on the board play an important role in solving the problem of long-range lidars of decimeter resolution. Such devices are now widely used in automotive and aircraft manufacturing, shipbuilding, in the field of optical location, automatic recognition systems, vision systems, and various fields of electronic engineering.

For pumping high-power wideband laser diodes used in laser lidars and other systems, there is a need to generate current pulses with a duration of several nanoseconds and an amplitude of $\sim 10 \div 100 \mathrm{~A}[1,2]$. In our opinion, the best available solution to this problem is associated with the use of high-voltage $(\sim 300 \mathrm{~V})$ avalanche transistors [3] for

The work was performed as part of the government assignment of the Ministry of Education and Science of the Russian Federation, project 11.5861.2017. optical pulses with a duration of 3 to $10 \mathrm{~ns}$ from 10 to $100 \mathrm{~W}$ laser diodes. Such an optical pulse duration becomes a limitation for lidars of high (decimeter) accuracy, if the task is to maximize the distance of the distance measurement (up to several kilometers or more) $[4,5]$. Indeed, the frequency range of the receiving channels based on avalanche detectors currently exceeds $300 \mathrm{MHz}$, which makes it possible to detect optical pulses with a duration of 1 ns without a noticeable decrease in the sensitivity of the detector and going beyond the critical limit of the noise level. Therefore, the development of a laser diode transmitter capable of emitting optical pulses with a duration of about $1 \mathrm{~ns}$ with the highest peak power (several tens of $\mathrm{W}$ ) becomes very relevant for long-range high-precision radars. The main problem is nanosecond high-current drivers. High-current $(10-100$ A) nanosecond, sub-nanosecond, and even picosecond drivers are on the market [6], but the high price and large size make it very difficult to use them in real conditions.

\section{EXPERIMENT AND DISCUSSION}

Previously, we proposed the design of a miniature nanosecond emitter for optical radar [7]. The schematic diagram of the radiator is shown in Fig. 1. The following simplest scheme allows to achieve the minimum duration and maximum amplitude of the current pulse for a given electrical switch. This circuit can be used in transmitters, replacing the load resistor with a laser diode.

The following simple but informative equations demonstrate the relationship between peak current amplitude $\mathrm{I}_{\mathrm{m}}$ and full width at half height (FWHM) of a pulse $\mathrm{t}_{\mathrm{w}}$ calculated for an "ideal" switch with infinitely small switching time between the levels U0 (maximum voltage on the switch) and $U_{R}$ (residual switch voltage):

$$
\begin{gathered}
t_{w}=2.2 \times \sqrt{L_{P} \times C_{0}} \\
I_{m}=\frac{U_{0}-U_{R}}{\sqrt{\frac{L_{P}}{C_{0}}}+R_{L}}
\end{gathered}
$$

Assuming the switch is ideal $\left(\mathrm{U}_{\mathrm{R}}=0\right.$, infinitely short switching time), and the load resistor is replaced by a laser diode with zero impedance, the following "quality criterion" of the transmitter can be derived: 


$$
I_{m} / t_{w}=\frac{U_{0}}{2.2 \times L_{P}}
$$

Obviously, for long-range high-precision radars, the highest quality criterion $\mathrm{I}_{\mathrm{m}} / \mathrm{t}_{\mathrm{w}}$ is necessary, and therefore the greatest possible offset $\mathrm{U}_{0}$ and the smallest possible inductance are preferable.

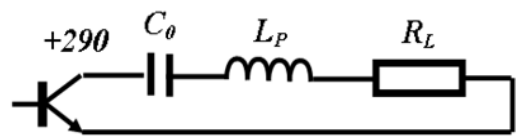

Fig. 1. The simplest and most efficient driver of the smallest current, which can be implemented in a miniature layout. The switch is represented by a bipolar transistor operating in the avalanche breakdown mode; the capacitor $\mathrm{C}_{0}$ accumulates the energy between the pulses (it is charged) and discharges through the load resistor $\mathrm{R}_{\mathrm{L}}$ and the total parasitic inductance of the circuit of the $L_{P}$ circuit. In the optical transmitter, the load resistor is replaced by a laser diode

The bias voltage is determined on the basis of a compromise between the above criteria, the switching speed of a particular transistor and the maximum voltage that a particular user allows for his system. The parasitic inductance $\mathrm{L}_{P}$ in all cases should be reduced, and its base limit is determined by the size of the circuit components shown in Fig. 1. Naturally, the layout should be implemented in the form of a three-dimensional structure of the minimum size, consisting of semiconductor chips [7].

When measuring the distance to the object, there is a variation in the results of single measurements and accurate determination of the distance requires averaging the results. Accuracy increases as the square root of the number of measurements. Operation of the radar in real time requires a fairly high frequency of repetition measurements. For example, at a frequency of $1 \mathrm{kHz}$, the measurement accuracy improves by about thirty times with a measurement time of 1 $\mathrm{s}$, and $10 \mathrm{kHz}$ will provide a hundred-fold improvement in measurement accuracy. The main factor limiting the frequency of the avalanche switch operation is that when switching there is a highly localized heating region and the permissible pulse repetition rate is determined by the time of thermal diffusion from this micron region to the heat sink.

In the previously developed modules [7], this time is about $1 \mathrm{~ms}$ and the guaranteed frequency of the module is not more than $0.5 \mathrm{kHz}$.

It was necessary to solve the problem of a significant increase in frequency, at least up to $10 \mathrm{kHz}$.

In Fig. 2 a switch assembly made on the basis of the above requirements, using FMMT491 transistors, LCC laser diodes, and specially designed surface-mounted chip capacitors is shown [8]. The assembly is made on the basis of the requirements of the minimum possible distance between the emitter of the transistor and the laser diode to ensure the minimum parasitic inductance, which limits the switching time.

On the basis of actual situation, one may say that none of the industrial capacitors on the market is fully suitable for high-power nanosecond laser transmitters due to poor dielectric quality or high parasitic inductance associated with large geometrical dimensions that do not minimize the parasitic inductance of the radiator design.

Fig. 3 shows a photograph of a laboratory sample of a fully assembled test module.

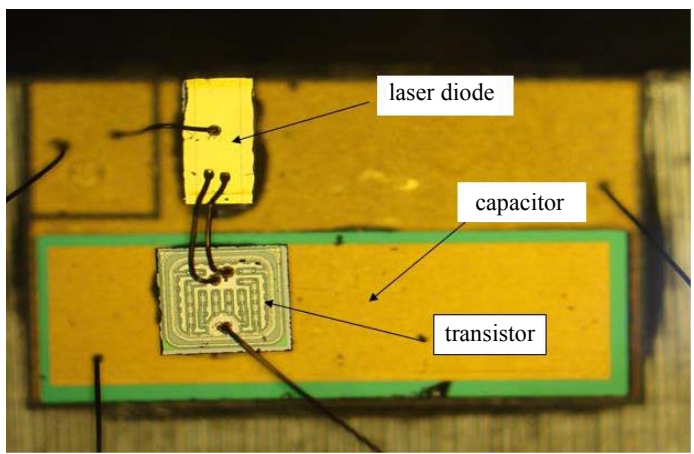

Fig. 2. Original switch assembly with a transistor mounted on a capacitor to ensure minimum parasitic inductance

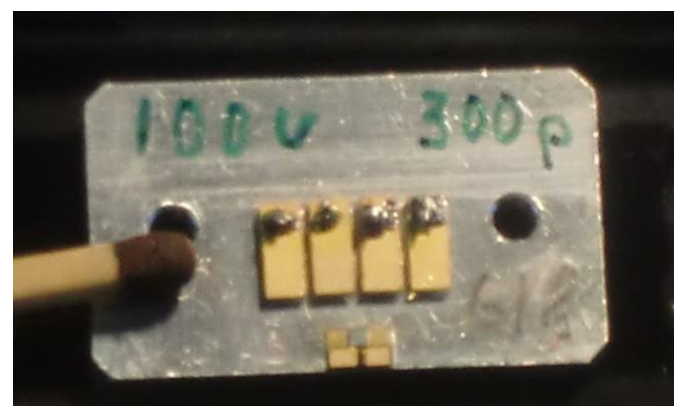

Fig. 3. Laboratory sample of the complete optical emitter module

The experimental study results of the possibility of reducing the duration and increasing the amplitude of a current pulse are shown in Fig. 4. The curves reflect the results of calculations obtained using formulas (1) and (2). The dashed curve is compared with the experimental results obtained by measuring the FMMT415 transistor, the continuous curve reflects the expected values for a switch with an infinitely small switching time, a residual voltage of $70 \mathrm{~V}$ and an inductance of $2.5 \mathrm{nH}$.

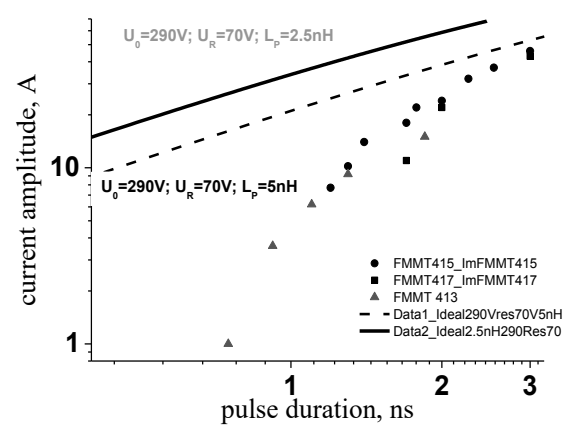

Fig. 4. Dependence of the peak current on the pulse duration, measured using multilayer $\mathrm{NP}_{0}$ ceramic capacitors of various capacities, a circuit with a total parasitic inductance of $5 \mathrm{nH}$ and various industrial avalanche transistors of models FMMT 415, 413 and 417 (manufactured by Zetex Semiconductors)

These rather approximate but illustrative calculations show the main limitations when trying to generate shorter pulses with an increased amplitude. Of course, the first step is to reduce the parasitic inductance, making changes to the layout. Further, the main limitation is the switching speed of the avalanche transistor [8].

The calculation curve for an ideal switch (with an infinitely small switching time) at $\mathrm{L}_{P}=5 \mathrm{nH}$ is indicated by a dotted line. It gives slightly larger values of $\mathrm{I}_{\mathrm{m}} / \mathrm{t}_{\mathrm{w}}$, but still comparable with the experimental data with FMMT415, as 
well as high $\mathrm{C}_{0}$ values (with a pulse duration of $\mathrm{t}_{\mathrm{w}}$, exceeding 2-3 ns). This means that when the switching time of the transistor is close to $2 \mathrm{~ns}$, the amplitude and duration of the current pulse, approximately calculated by equations (1) and (2), do not differ too much from the experimental results, while for shorter pulses, the differences increase noticeably.

It has been established that the main factor limiting the frequency range of the avalanche optical switch operation is the mechanism of high-frequency degradation. Immediately after a single switch, the temperature in the hot region (crosssection of a few microns) located at the collectorsubcollector boundary reaches $700-800 \mathrm{~K}$. Cooling through a 200 micron thick substrate occurs with a characteristic thermal diffusion time of a few tenths of a millisecond $(\sim 0.4$ $\mathrm{ms}$ ). This means that a sufficiently complete cooling, which prevents the gradual accumulation of heat from pulse to pulse, requires several such time constants $(>1 \mathrm{~ms})$, and therefore $1 \mathrm{kHz}$ is the upper limit of the allowable operating frequency range of the radiator. If the next pulse arrives earlier than several time constants of thermal diffusion, heat accumulation in the breakdown region begins, heat accumulation in the hot region, which after some time (about $10^{5}$ pulses) will bring the temperature in this region to a thermal destruction of silicon near the collector-subcollector boundary.

Even earlier, the degradation of the device arises due to the thermal cord, which arose as a result of the following process. After switching the device, the voltage on it will begin to increase almost from zero, tending to reach the power supply voltage in ms or a bit earlier. As a result, on the still not cooled device, voltage will begin to be applied to the "spreading out" and gradually cooling down hot area, while the carriers in the conducting channel have not recombined yet. Consequently, additional heating of the entire channel will begin, preventing the hot area from complete cooling. With a certain set of parameters, this process will lead to the formation of a thermal cord, and that, in turn, to the destruction of the device. Note that the maximum temperature is expected near the $n^{0}-n^{+}$boundary of the collector, which will primarily lead to destruction of the collector layer, somewhat remote from the $p-n$ junction, and we can expect not a complete transistor shorting, but a reduction in the breakdown voltage to a value determined by the thickness of the intact part $\mathrm{n}^{0}$ - collector layer.

Since it is currently not possible to obtain transistor crystals with a thinned substrate, in this work the problem of the switch operation reliability at frequencies up to $10 \mathrm{kHz}$ is solved by other methods - the additional mechanism of heating the hot zone and the switching channel between nanosecond pulses is eliminated. For charging the capacity, the transistor is connected to a power source of the order of hundreds of volts through a certain resistance $\mathrm{R}$ (of the order of several tens of $\mathrm{kOhms}$ ), and the hot area and the avalanche current passageway begin to heat up due to the product of the current (decreasing in time from $\mathrm{V}_{0} / \mathrm{R}$ to zero, where $\mathrm{V}_{0}$ is the voltage of the power source) for voltage (increasing from zero to $\mathrm{V}_{0}$ ). The current flows due to the fact that the carriers in the switching channel have not yet become extinct by the time when the voltage on the structure began to increase, and in addition there are carriers created by thermal generation in the hot zone. To eliminate this additional warm-up, we have done the following. Before the moment of switching, the transistor is disconnected from the voltage source schematically, and after a nanosecond current pulse, spherical heat spreading from the hot region and extinction of nonequilibrium carriers begin. As a result, before the arrival of the next launch pulse, all the carriers died out, and the hot zone managed to cool down significantly (as far as thermal diffusion through the substrate allowed). After that, the capacitance connected to the collector of the transistor is charged by the active charging circuit to voltage $V_{0}$, charging is turned off, and the next avalanche transistor starts. This approach does not eliminate, of course, the problem of cooling the hot area through the substrate, but eliminates the very undesirable mechanism of additional heating, which made it possible to increase the operating frequency by almost an order of magnitude.

\section{CONCLUSION}

We have developed a switch board assembly method, which allows a strong reduction in thermal diffusion time while keeping the parasitic inductance of the capacitor discharge circuit at its minimum.

An optimal combination of transistor type and corresponding operating voltage has been determined experimentally.

High-frequency degradation mechanism has been identified as the main factor limiting the operating frequency range of the avalanche optical switch.

To overcome the problem of overheating of the avalanche switch a specific power supply scheme has been developed, in which the charging of capacitor is allowed only after the device is sufficiently cooled down after switching.

A further increase in the frequency is possible under the condition of reducing the thickness of the transistor crystal. Reducing the thickness leads to a quadratic dependence of the operating frequency of the switching of the optical driver, that is, thinning the substrate two to three times would increase the frequency four to nine times, and reducing the distance from $\mathrm{n} 0-\mathrm{n}+$ collector boundary to the heat sink using special technological methods to $\sim 10$ um would allow to increase the operating frequency to at least $0.1-1 \mathrm{MHz}$.

\section{REFERENCES}

[1] A. Biernat, G. Kompa. Powerful picosecond laser pulses enabling high - resolution pulsed laser radar. J. Optics. 1998. Vol. 29. P. 225228

[2] A. Kilpela, J. Kostamovaara. Laser pulser for a time-of-flight laser radar. Rev. Sci. Instr. 1997. Vol.68. P. 2253-2258

[3] S. N. Vainshtein, G. Duan, A. V. Filimonov, J. T. Kostamovaara. Switching mechanisms triggered by a collector voltage ramp in avalanche transistors with short-connected base and emitter. IEEE Transactions on Electron Devices. 2016. Vol. 63. Iss. 8. P. 3044 3048

[4] W. B. Herden, "Application of avalanche transistors to circuits with a long mean time to failure", IEEE Trans. Instrum. Meas., vol. IM-25, no. 2, 740 pp. $152-160,1976$.

[5] J.M.T. Huikari, E.A. Avrutin, B.S. Ryvkin, J.J. Nissinen, and J.T. Kostamovaara, "High-Energy picosecond pulse generation by gain switching in asymmetric waveguide structure multiple quantum well lasers", IEEE J. Sel. Topics Quantum Electron., vol. 21, no. 6, 2015, Art. no. 7621501206

[6] FID GmbH website: http://www.fidtechnology.com

[7] Filimonov A.V., Zemlyakov V.E., Egorkin V.I., Maslevtsov A.V., Wurz M.C., Vainshtein S.N. Nanosecond miniature transmitters for pulsed optical radars. Lecture Notes in Computer Science. Vol. 10531 LNCS. 2017. P. 490-497

[8] S. Vainshtein, V. Zemlyakov, V. Egorkin, A. Maslevtsov, A. Filimonov. Miniature high-power nanosecond laser diode transmitters using the simplest possible avalanche drivers // IEEE Transactions on Power Electronics. 2019. Vol. 34. Iss. 4. P. 3689-3699. 